\title{
Vector bundles on a neighborhood of an exceptional curve and elementary transformations
}

\author{
E. Ballico - E. Gasparim*
}

\begin{abstract}
Let $W$ be the germ of a smooth complex surface around an exceptional curve and let $E$ be a rank 2 vector bundle on $W$. We study the cohomological properties of a finite sequence $\left\{E_{i}\right\}_{1 \leq i \leq t}$ of rank 2 vector bundles canonically associated to $E$. We calculate numerical invariants of $E$ in terms of the splitting types of $E_{i}, 1 \leq i \leq t$. If $S$ is a compact complex smooth surface and $E$ is a rank two bundle on the blow-up of $S$ at a point, we show that all values of $c_{2}(E)-c_{2}\left(\pi_{*}(E)^{* *}\right)$ that are numerically possible are actually attained.
\end{abstract}

2000 Mathematics Subject Classification: 14F05

\section{Introduction}

We consider exceptional curves in the following two cases. In the first case, let $\mathrm{W}$ be a smooth connected complex analytic surface which contains an exceptional divisor i.e. a smooth curve $\mathrm{D} \cong \mathbf{P}^{1}$ with $\boldsymbol{O}_{\mathrm{D}}(-1)$ as normal bundle. Let $\mathrm{U}$ be a small tubular neighborhood of $D$ in the Euclidean topology and let $\pi: U \rightarrow Z$ be the contraction of $D$. In this case $\mathrm{Z}$ is the germ of a smooth surface around the point $\mathrm{P}:=\pi(\mathrm{D})$.

In the second case, let $\mathrm{W}$ be a smooth connected algebraic surface defined over an algebraically closed field $\mathbf{K}$ with arbitrary characteristic. We assume that $\mathbf{W}$ contains an exceptional curve $\mathrm{D}$ and denote by $\mathrm{U}$ the formal completion of $\mathrm{W}$ along $\mathrm{D}$. Let $\pi$ : $U \rightarrow Z$ be the contraction of $\mathrm{D}$. In this case $\mathrm{Z}$ is a formal smooth two-dimensional space supported at $\mathrm{P}$.

In what follows we use the notation defined above to represent either case. Let I be the ideal sheaf of $\mathrm{D}$ in $\mathrm{U}$ and consider a rank 2 vector bundle $\mathrm{E}$ over $\mathrm{U}$. Consider the pair of integers $(\mathrm{a}, \mathrm{b})$ such that $\mathrm{E} \mid \mathrm{D} \cong \boldsymbol{O}_{\mathrm{D}}(\mathrm{a}) \oplus \boldsymbol{O}_{\mathrm{D}}(\mathrm{b})$. We will refer to the pair $(\mathrm{a}, \mathrm{b})$ as the splitting type of $\mathrm{E}$. Since $\mathrm{Z}$ is a smooth surface the bidual $\pi_{*}(\mathrm{E})^{* *}$ is locally free and hence free because $\mathrm{Z}$ is 2 -dimensional. There is a natural inclusion $\mathbf{j}: \pi_{*}(\mathrm{E}) \rightarrow \pi_{*}(\mathrm{E})^{* *}$ such that coker $(\mathbf{j})$ has finite length. Set $Q:=\operatorname{coker}(\mathbf{j})$.

We show that the pair $(\mathrm{z}, \mathrm{w}):=\left(\mathrm{h}^{0}(\mathrm{Z}, Q), \mathrm{h}^{0}\left(\mathrm{Z}, \mathrm{R}^{1} \pi_{*}(\mathrm{E})\right)\right)$ of numerical invariants of $\mathrm{E}$ is uniquely determined by a sequence of pairs of integers associated to $E$ in $[\mathbf{B}]$ using ele-

\footnotetext{
* The first author was partially supported by MURST (Italy) and the second author was partially supported by NSF grant DMS0072675 .
} 
mentary transformations. We review the construction of the associated sequence and prove the following results.

Theorem 0.1. Let $E$ be a rank 2 vector bundle on $W$ with associated admissible sequence $\left\{\left(a_{i}, b_{i}\right)\right\}_{1} \leq i \leq t$. Then we have the equalities

$$
\begin{aligned}
& w:=h^{0}\left(Z, R^{1} \pi_{*}(E)\right)=\Sigma_{1 \leq i \leq t} \max \left\{-b_{i^{-}} 1,0\right\} \quad \text { and } \\
& z:=h^{0}(Z, Q)=\Sigma_{1 \leq i \leq t} a_{i}-a t^{2}-\Sigma_{1 \leq i \leq t} \max \left\{-b_{i}-1,0\right\} .
\end{aligned}
$$

Every admissible sequence is associated to a rank 2 vector bundle on $\mathrm{W}$ (see [B] Th.0.2). For simplicity, we normalize our bundles to have splitting type $(j,-j+\varepsilon)$, with $\varepsilon=0$ or $\varepsilon=-1$. We have the following existence theorem.

Theorem 0.2. For every pair of integers $(z, w)$ satisfying $j-1-\varepsilon \leq w \leq j(j-1) / 2-j \varepsilon$ and $1 \leq$ $z \leq j(j+1) / 2$ with $j \geq 0$ and $\varepsilon=0$ or -1 , there exists a rank 2 vector bundle $E$ on $W$ with splitting type $(j,-j+\varepsilon)$ having numerical invariants $h^{0}\left(Z, R^{l} \pi_{*}(E)\right)=w$ and $h^{0}(Z, Q)=z$.

Remark 0.3. It follows from theorem 0.2 that the strata defined in [BG] for spaces of bundles on the blow-up of $\boldsymbol{C}^{2}$ are all non-empty.

We give also the following characterization of the split bundle.

Proposition 0.4. Let $E$ be a rank 2 vector bundle on $U$ with splitting type $(j,-j+\varepsilon)$ with $j>0$ and $\varepsilon=0$ or -1 . The following conditions are equivalent:

(i) $\quad E \cong \boldsymbol{O}_{U}(-j D) \oplus \boldsymbol{O}_{U}((j+\varepsilon) D)$

(ii) $\quad c_{2}(E)-c_{2}\left(\pi_{*}(E)^{* *}\right)=j(j+\varepsilon)$

(iii) $\quad h^{0}\left(Z, R^{l} \pi_{*}(E)\right)=j(j-1) / 2$

(iv) E has associated sequence $\left\{\left(a_{i}, b_{i}\right)\right\}_{1} \leq i \leq j-\varepsilon$ with $b_{i}=-j-\varepsilon+i-1$ for every $i$.

We now consider a compact complex smooth surface $S$, so that we can calculate second chern classes. If $\mathrm{E}$ is a rank 2 bundle defined on the blow-up of $\mathrm{S}$ at a point, then the difference of second Chern classes satisfies $j \leq c_{2}(E)-c_{2}\left(\pi_{*}(E)^{* *}\right) \leq j^{2}$ and is given by the sum $h^{0}\left(Z, R^{1} \pi_{*}(E)\right)+h^{0}(Z, Q)$ (see [FM]). Sharpness of these bounds was proven in [B] and in [G2] by different methods. We prove the following existence theorem. 
Theorem 0.5. Let $\boldsymbol{S}$ be the blow-up of a compact complex smooth surface $S$ at a point. Let $l$ denote the exceptional divisor and let $j$ be a non-negative integer. Then for every integer $k$ satisfying $j \leq k \leq j^{2}$ there exists a rank 2 vector bundle $\mathrm{E}$ over $\mathbf{S}$ with $\mathrm{E} \mid l \cong \boldsymbol{O}_{l}(\mathrm{j})$

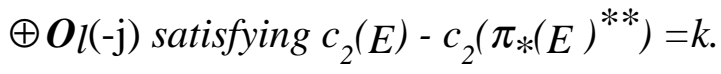

Note 0.6: In [G1, Thm. 3.5] it is shown that the number of moduli for the space of rank-2 bundles on the blow up of $\mathbf{C}^{2}$ at the origin with splitting type $\mathrm{j}$ equals $2 \mathrm{j}-3$; and since such bundles are determined by their restriction to a formal neighborhood of the exceptional divisor it follows that we have the same number of moduli for bundles over the neighborhood $\mathrm{U}$ of an exceptional curve on a surface $\mathrm{W}$.

These results are proven in section 1, where we also review the construction of admissible sequences. On section 2 we consider briefly bundles of higher rank.

\section{Rank 2 bundles}

We briefly recall the construction of the associated sequences of pairs of bundles and splitting types given in the introduction of $[\mathbf{B}]$. We first give the definitions of positive and negative elementary transformations.

Let $\mathrm{E}$ be a rank 2 vector bundle on $\mathrm{W}$ with splitting type $(\mathrm{a}, \mathrm{b})$ with $\mathrm{a} \geq \mathrm{b}$. Fix a line bundle $\mathrm{R}$ on $\mathrm{D}$ and a surjection $\mathbf{r}: \mathrm{E} \rightarrow \mathrm{R}$ induced by a surjection $\rho: \mathrm{E} \mid \mathrm{D} \rightarrow \mathrm{R}$. There exists such a surjection if and only if $\operatorname{deg}(R) \geq b$. If $\operatorname{deg}(R)=b<a$, then $\rho$ is unique, up to a multiplicative constant. Set $E^{\prime}:=\operatorname{ker}(\mathbf{r})$ and $R^{\prime}=\operatorname{ker}(\rho)$. If $\operatorname{deg}(R)=b<a$ the sheaf $E^{\prime}$ is uniquely determined, up to isomorphism. Since $\mathrm{D}$ is a Cartier divisor, $\mathrm{E}^{\prime}$ is a vector bundle on $\mathrm{U}$. We will say that $\mathrm{E}^{\prime}$ is the bundle obtained from $\mathrm{E}$ by making the negative elementary transformation induced by $\mathbf{r}$. Note that $\mathrm{R}^{\prime}$ is a line bundle on $\mathrm{D}$ with degree $\operatorname{deg}\left(R^{\prime}\right)=a+b-\operatorname{deg}(R)$. Since $\operatorname{deg}\left(\mathbf{I} / \mathbf{I}^{2}\right)=1$ it is easy to check that $\operatorname{deg}\left(E^{\prime} \mid D\right)=a+b+1$ and we have the exact sequence

$$
0 \rightarrow \boldsymbol{O}_{\mathrm{D}}(1+\operatorname{deg}(\mathrm{R})) \rightarrow \mathrm{E}^{\prime} \mid \mathrm{D} \rightarrow \mathrm{R}^{\prime} \rightarrow 0 .
$$

Furthermore, using this exact sequence we obtain a surjection $\mathbf{t}: \mathrm{E}^{\prime} \rightarrow \mathrm{R}^{\prime}$ such that $\operatorname{ker}(\mathbf{t})$ $\cong \mathrm{E}(-\mathrm{D})$. In particular $\operatorname{ker}(\mathbf{t}) \mid \mathrm{D} \cong \boldsymbol{O}_{\mathrm{D}}(\mathrm{a}+1) \oplus \boldsymbol{O}_{\mathrm{D}}(\mathrm{b}+1)$. Thus, up to twisting by $\boldsymbol{O}_{\mathrm{U}}(-\mathrm{D})$, the negative elementary transformation induced by $\mathbf{r}$ has an inverse operation and we will say that $\mathrm{E}$ is obtained from $\mathrm{E}$ ' making a positive elementary transformation supported by D. The following diagram, called the display of the elementary transformation, summarizes the construction (see $[\mathbf{M}]$ ). 


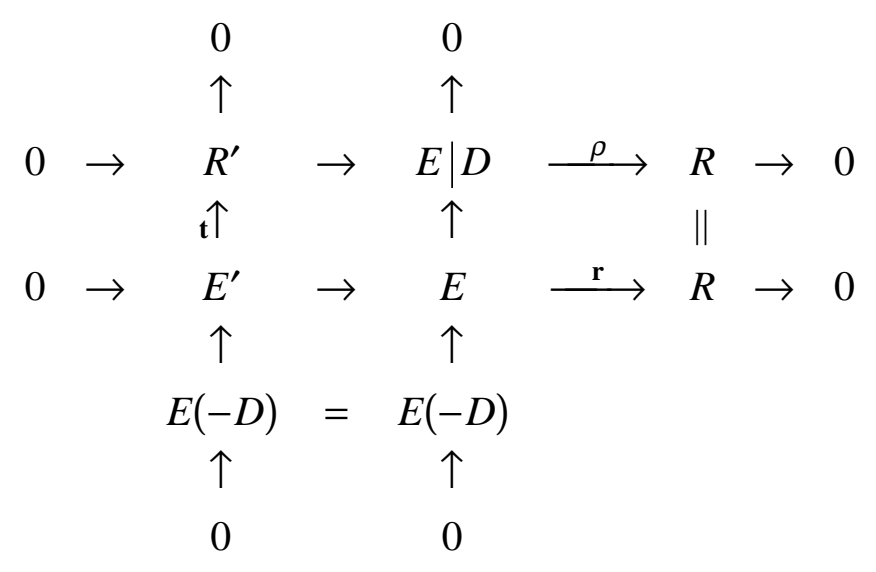

Given two vector bundles $\mathrm{E}_{1}$ and $\mathrm{E}_{2}$ with splitting types $\left(a_{1}, b_{1}\right)$ and $\left(a_{2}, b_{2}\right)$ we say that $\mathrm{E}_{1}$ is more balanced then $\mathrm{E}_{2}$ if $a_{1}-b_{1} \leq a_{2}-b_{2}$. Given a vector bundles $\mathrm{E}$ with splitting type $(a, b)$ we say that $\mathrm{E}$ is balanced if either $\mathrm{a}=\mathrm{b}$ (case $c_{1}$ even) or else $\mathrm{a}=\mathrm{b}+1$ (case $c_{1}$ odd). Performing negative elementary transformations we will take the bundle $\mathrm{E}$ into more balanced bundles. The sequence of elementary transformations finishes when we arrive at a balanced bundle. If $\operatorname{deg}(R)=b$, then $E^{\prime} \mid D$ fits in the exact sequence

$$
0 \rightarrow \boldsymbol{O}_{\mathrm{D}}(\mathrm{b}+1) \rightarrow \mathrm{E}^{\prime} \mid \mathrm{D} \rightarrow \boldsymbol{O}_{\mathrm{D}}(\mathrm{a}) \rightarrow 0
$$

If $\mathrm{b}<\mathrm{a}$ then $\mathrm{E}^{\prime}$ is more balanced than $\mathrm{E}$. If $\mathrm{b} \leq \mathrm{a}-3$, then (2) does not uniquely determine $E^{\prime} \mid C$. If $b \leq a-2$ and $E^{\prime}$ is not balanced, we reiterate the construction starting from $E^{\prime}$ taking $R^{\prime}$ to be the factor of $E^{\prime} \mid D$ of lowest degree and we take the unique surjection (up to a multiplicative constant) $\rho^{\prime}: E^{\prime} \rightarrow R^{\prime}$. In a finite number, say, $t-1$, of steps, we send $E$ into a bundle which, up to twisting by $\boldsymbol{O}_{\mathrm{U}}(\mathrm{sD})$, where $\mathrm{s}=-(\mathrm{a}+\mathrm{b}+\mathrm{t}-1) / 2$ has trivial restriction to D. The process ends with a bundle isomorphic to $\boldsymbol{O}_{\mathrm{U}}(\mathrm{sD})^{\oplus 2}$ (see [B], Remark 0.1).

We now construct the admissible sequence associated to $E$. Step one: set $E_{1}:=E, a_{1}:=$ $a$ and $b_{1}:=b$. If $a_{1}=b_{1}$, set $t=1$ and stop. Otherwise $a_{1}>b_{1}$. Step two: in the case $a_{1}>b_{1}$ set $E_{2}:=E^{\prime}$ and let $\left(a_{2}, b_{2}\right)$ be the splitting type of $E^{\prime}$. Note that $a_{2}+b_{2}=a_{1}+b_{1}+1$ and $b_{1}$ $<b_{2} \leq a_{2} \leq a_{1}$. Hence $a_{2}-b_{2}<a_{1}-b_{1}$ and $E_{2}$ is more balanced than $E_{1}$. If $a_{2}=b_{2}$, set $t:=2$ and stop. If $a_{2}>b_{2}$ reiterate the construction. Final step: in a finite number of steps (say $t$ -1 steps) we arrive at a bundle $E_{t}$ with splitting type (at, $\left.b_{t}\right)$ with $a t=b_{t}$. Call $E_{i}, 2 \leq i \leq t$, the bundle obtained after $\mathrm{i}-1$ steps and let $\left(\mathrm{a}_{\mathrm{i}}, \mathrm{b}_{\mathrm{i}}\right)$ be the splitting type of $\mathrm{E}_{\mathrm{i}}$. The finite sequence of pairs $\left\{\left(a_{i}, b_{i}\right)\right\} \quad 1 \leq \mathrm{i} \leq \mathrm{t}$ obtained in this way has the following properties:

$$
\begin{array}{ll}
\text { i. } & a_{i} \geq b_{i} \forall i>0, \\
\text { ii. } & a_{i}+b_{i}=a_{1}+b_{1}+i-1 \forall i>1, \\
\text { iii. } & a_{i} \geq a_{i}+1 \geq b_{i}+1>b_{i} \forall i \geq 1, \text { and } \\
\text { iv. } & a_{t}=b_{t} .
\end{array}
$$


We call admissible any such finite sequence of pairs of integers. We will say that a sequence $\left\{\left(\mathrm{a}_{\mathrm{i}}, \mathrm{b}_{\mathrm{i}}\right)\right\}, 1 \leq \mathrm{i} \leq \mathrm{t}$ is the admissible sequence associated to the bundle $E$ if this sequence is created by the algorithm just described. By [B] Th. 0.2, every admissible sequence is associated to a rank 2 vector bundle on $\mathrm{W}$.

Examples: Let us first set some notation. To represent the admissible sequence $\left\{\left(\mathrm{a}_{\mathrm{i}}, \mathrm{b}_{\mathrm{i}}\right)\right\}, 1 \leq \mathrm{i} \leq \mathrm{t}$, we write $\left(\mathrm{a}_{1}, \mathrm{~b}_{1}\right) \rightarrow\left(\mathrm{a}_{2}, \mathrm{~b}_{2}\right) \rightarrow \ldots \rightarrow\left(\mathrm{a}_{\mathrm{t}}, \mathrm{b}_{\mathrm{t}}\right)$.

1. If the splitting type of $E$ is $(b+2, b)$ then there is only one possibility for the admissible sequence associated to E, namely

$$
(b+2, b) \rightarrow(b+2, b+1) \rightarrow(b+2, b+2)
$$

2. If the splitting type of $\mathrm{E}$ is $(\mathrm{b}+4, \mathrm{~b})$ then there are 3 different possibilities for admissible sequences associated to $\mathrm{E}$ (which in particular will give rise to different values of the numerical invariants $(\mathrm{z}, \mathrm{w}))$, these are:

$$
\begin{array}{ll}
\text { i. } & (b+4, b) \rightarrow(b+4, b+1) \rightarrow(b+4, b+2) \rightarrow(b+4, b+3) \rightarrow(b+4, b+4) \\
\text { ii. } & (b+4, b) \rightarrow(b+4, b+1) \rightarrow(b+3, b+3) \\
\text { iii. } & (b+4, b) \rightarrow(b+3, b+2) \rightarrow(b+3, b+3)
\end{array}
$$

We now calculate the numerical invariants of $E$ in terms of admissible sequences. For every integer $n \geq 0$ let $D^{(n)}$ be the $n$-th infinitesimal neighborhood of $D$ in $U$. Hence $D^{(n)}$ is the closed subscheme of $U$ with $\mathbf{I}^{\mathrm{n}+1}$ as ideal sheaf. In particular, $\mathrm{D}^{(0)}=\mathrm{D}$ and $D^{(n)}$ red $=D$ for every $n \geq 0$. For each integer $n \geq 0$ the following sequence is exact

$$
0 \rightarrow \mathbf{I}^{\mathrm{n}} / \mathbf{I}^{\mathrm{n}+1} \rightarrow \boldsymbol{O}_{\mathrm{U}} / \mathbf{I}^{\mathrm{n}+1} \rightarrow \boldsymbol{O}_{\mathrm{U}} / \mathbf{I}^{\mathrm{n}} \rightarrow 0
$$

Suppose that $E$ is a vector bundle normalized to have splitting type $(j,-j+\varepsilon)$ where $j \geq 1$ and either $\varepsilon=0$ or $\varepsilon=-1$. We denote by $\mathbf{m}$ be the maximal ideal of $\boldsymbol{O} Z$,P. Consider the inclusion $\mathbf{j}: \pi_{*}(\mathrm{E}) \rightarrow \pi_{*}(\mathrm{E})^{* *}$ and let $Q:=\operatorname{coker}(\mathbf{j}), \mathrm{z}:=\mathrm{h}^{0}(\mathrm{Z}, Q)$, and $\mathrm{w}:=\mathrm{h}^{0}\left(\mathrm{Z}, \mathrm{R}^{1} \pi_{*}(\mathrm{E})\right)$. Call $\boldsymbol{O}_{\mathrm{D}}(\mathrm{x})$ the degree $\mathrm{x}$ line bundle on D. Twisting the exact sequence (3) by E and using the fact that $\mathbf{I}^{\mathrm{n}} / \mathbf{I}^{\mathrm{n}+1}$ has degree $\mathrm{n}$, we obtain the exact sequence

$$
0 \rightarrow \boldsymbol{O}_{\mathrm{D}}(\mathrm{j}+\mathrm{n}) \oplus \boldsymbol{O}_{\mathrm{D}}(-\mathrm{j}+\boldsymbol{\varepsilon}+\mathrm{n}) \rightarrow \mathrm{E}|\mathrm{D}(\mathrm{n}) \rightarrow \mathrm{E}| \mathrm{D}(\mathrm{n}-1) \rightarrow 0
$$

Lemma 1.1. The integers $z$ and $w$ satisfy the inequalities:

$$
1 \leq z \leq j(j+1) / 2 \quad \text { and } \quad j-1-\varepsilon \leq w \leq j(j-1) / 2-\varepsilon j .
$$


Proof. By the Theorem on Formal Functions we have the bounds for $\mathrm{z}$ and we have that $\mathrm{w} \leq \Sigma_{\mathrm{n}} \geq 0 \mathrm{~h}^{1}\left(\mathrm{D}, \boldsymbol{O}_{\mathrm{D}}(-\mathrm{j}+\varepsilon+\mathrm{n})\right)=\mathrm{j}(\mathrm{j}-1) / 2-\varepsilon \mathrm{j}$. The upper bound for $\mathrm{w}+\mathrm{z}$ was stated in $[\mathbf{F M}]$ Remark 2.8, and proven for bundles with arbitrary rank in [Bu] Prop.2.8. Consequently we have an alternative proof of the upper bound for $\mathrm{z}$. The lower bound for $\mathrm{w}$ will be proven in Remark 1.4. For the case of rank two and $\varepsilon=0$ [G2] shows that these bounds are sharp.

Since $Q$ is a quotient of $\boldsymbol{O} \mathrm{U}, \mathrm{P}^{\oplus 2}$ the dimension of the fiber of $Q$ at $\mathrm{P}$ is either 1 or 2 . The sheaf $Q$ is isomorphic to the structure sheaf of a subscheme of $\mathrm{Z}$ supported by $\mathrm{P}$ and with length $\mathrm{z}$ if and only if the dimension of this fiber is 1 . We will check that this is always true (see Proposition 1.3). We first check the split case.

Lemma 1.2. Suppose that $E \cong \boldsymbol{O}_{U}(-j D) \oplus \boldsymbol{O}_{U}((j-\varepsilon) D)$ then $z=j(j+1) / 2, w=j(j-1) / 2-\varepsilon j$ and $Q$ is isomorphic to the structure sheaf of a subscheme of $Z$ supported by $P$ and with $\boldsymbol{m} j$ as ideal sheaf.

Proof. Since D is an exceptional divisor, we have $\pi_{*}\left(\boldsymbol{O}_{\mathrm{U}}((\mathrm{j}-\varepsilon) \mathrm{D})\right)=\pi_{*}\left(\boldsymbol{O}_{\mathrm{U}}\right)=\boldsymbol{O}_{\mathrm{Z}}$ for every $\mathrm{j} \geq \varepsilon$ and $\pi_{*}\left(\boldsymbol{O}_{\mathrm{U}}(-\mathrm{jD})\right) \cong \mathbf{m} \mathrm{j}$ if $\mathrm{j}>0$.

Proposition 1.3. Let $E$ be a rank 2 vector bundle on $W$ with splitting type $(j,-j+\varepsilon)$ with $j>$ 0 . Then $Q$ is isomorphic to the structure sheaf of a length $z$ subscheme $Q$ of $Z$ with $Q_{\text {red }}$ $=P$ and $Q \subseteq P(j-1)$.

Proof of 1.3. The first assertion is well-known and follows from the proof of Lemma 1.2. Since $Q$ is a quotient of $\boldsymbol{O}_{\mathrm{Z}}^{\oplus 2}$, in order to prove the second assertion it is sufficient to check that its fiber at $P$ is a 1 -dimensional vector space. Since $E$ has splitting type $(j,-j+\varepsilon)$, we have an extension

$$
0 \rightarrow \boldsymbol{O}_{\mathrm{U}}((-\mathrm{j}+\varepsilon) \mathrm{D}) \rightarrow \mathrm{E} \rightarrow \boldsymbol{O}_{\mathrm{U}}(\mathrm{jD}) \rightarrow 0
$$

([BG] Lemma 1.2, or in [G1] Thm. 2.1 in the case $\varepsilon=0$ ). Call e the extension (5) giving E. For each $t \in \mathbf{K} \backslash\{0\}$ consider the extension of $\boldsymbol{O}_{\mathrm{U}}(\mathrm{jD})$ by $\boldsymbol{O}_{\mathrm{U}}((-\mathrm{j}+\varepsilon) \mathrm{D})$ given by extension class te, this extension has as middle term a vector bundle isomorphic to E. Using the extension e for $t=0$, we construct a family $\{\lambda \mathbf{e}\} \lambda \in \mathbf{K}$ of extensions. We call $E_{\lambda}$ the corresponding middle term and $Q_{\lambda}$ the corresponding sheaf. Since $\mathrm{E}_{\lambda} \cong \mathrm{E}$ for $\lambda \neq 0$, we have $Q_{\lambda}=Q$ for $\lambda \neq 0$, and because $\mathrm{E}_{0} \cong \boldsymbol{O}_{\mathrm{W}}(\mathrm{jD}) \oplus \boldsymbol{O}_{\mathrm{W}}((-\mathrm{j}+\varepsilon) \mathrm{D})$, we have that $Q_{0}=\mathrm{P}(\mathrm{j}-1)$, and the result follows by semi-continuity of the fiber dimension at $\mathrm{P}$.

Proof of 0.1.Given the admissible sequence of splitting types $\left\{\left(\mathrm{a}_{\mathrm{i}}, \mathrm{b}_{\mathrm{i}}\right)\right\}_{1 \leq \mathrm{i} \leq \mathrm{t}}$ associated to E we want to show that 


$$
\begin{aligned}
& \mathrm{w}:=\mathrm{h}^{0}\left(\mathrm{Z}, \mathrm{R}^{1} \pi_{*}(\mathrm{E})\right)=\Sigma_{1 \leq \mathrm{i} \leq \mathrm{t}} \max \left\{-\mathrm{b}_{\mathrm{i}}-1,0\right\} \quad \text { and } \\
& \mathrm{z}:=\mathrm{h}^{0}(\mathrm{Z}, \mathrm{Q})=\Sigma_{1 \leq \mathrm{i} \leq \mathrm{t}} \mathrm{a}_{\mathrm{i}}-\mathrm{at}^{2}-\Sigma_{1 \leq \mathrm{i} \leq \mathrm{t}} \max \left\{-\mathrm{b}_{\mathrm{i}}-1,0\right\}
\end{aligned}
$$

We use induction on $\mathrm{t}$, the case $\mathrm{t}=1$ arising if and only if $\mathrm{a}_{1}=\mathrm{b}_{1}$, equivalently, when $\mathrm{E} \cong \boldsymbol{O}_{\mathrm{W}}\left(-\mathrm{a}_{1} \mathrm{D}\right)^{\oplus 2}$ (this follows immediately from the definition of admissible sequence). Since

$$
\begin{aligned}
& \mathrm{R}^{1} \pi_{*}\left(\boldsymbol{O}_{\mathrm{W}}(\mathrm{xD})\right)=0 \forall \mathrm{x} \leq 1 \text { and } \\
& \mathrm{R}^{1} \pi_{*}\left(\boldsymbol{O}_{\mathrm{W}}(\mathrm{yD})\right)=\mathrm{y}(\mathrm{y}-1) / 2 \forall \mathrm{y}>0
\end{aligned}
$$

we have the equality for $w$ in the split case. Assume $t \geq 2$. By the definition of the sequence $\left\{E_{i}\right\}_{1 \leq i \leq t}$ associated to $E$ we have that $E_{1}=E$ and that there is an exact sequence

$$
0 \rightarrow \mathrm{E}_{2} \rightarrow \mathrm{E}_{1} \rightarrow \boldsymbol{O}_{\mathrm{D}}\left(-\mathrm{b}_{1} \mathrm{D}\right) \rightarrow 0
$$

First assume $b_{1}<0$, in which case we have that $h^{0}\left(Z, \pi_{*}\left(\boldsymbol{O}_{\mathrm{D}}\left(-\mathrm{b}_{1} \mathrm{D}\right)\right)\right)=0$ and $\mathrm{h}^{0}\left(\mathrm{Z}, \mathrm{R}^{1} \pi_{*}\left(\boldsymbol{O}_{\mathrm{W}}\left(-\mathrm{b}_{1} \mathrm{D}\right)\right)\right)=-\mathrm{b}_{1}-1$. Hence $\mathrm{w}:=\mathrm{h}^{0}\left(\mathrm{Z}, \mathrm{R}^{1} \pi_{*}(\mathrm{E})\right)=\mathrm{h}^{0}\left(\mathrm{Z}, \mathrm{R}^{1} \pi_{*}\left(\mathrm{E}_{2}\right)\right)-\mathrm{b}_{1}+1$ and since $E_{2}$ has $\left\{\left(a_{i+1}, b_{i+1}\right)\right\} 1 \leq i<t$ as admissible sequence, the claim follows.

Now assume $b_{1} \geq 0$, from the exact sequence (6) it follows that $h^{0}\left(Z, R^{1} \pi_{*}(E)\right) \leq h^{0}\left(Z, R^{1}\right.$ $\left.\pi_{*}\left(E_{2}\right)\right)$. Since $b_{i}>b_{1}$ for every $i>1$, we have $h^{0}\left(Z, R^{1} \pi_{*}\left(E_{2}\right)\right)=0$. Hence, by the inductive assumption on the length of the admissible sequence, it follows that $\mathrm{h}^{0}\left(\mathrm{Z}, \mathrm{R}^{1} \pi_{*}(\mathrm{E})\right)=$ 0 , proving the first assertion. The value of $\mathrm{z}:=\mathrm{h}^{0}(\mathrm{Z}, Q)$ comes from the equalities $\mathrm{c}_{2}(\mathrm{E})-$ $\mathrm{c}_{2}\left(\pi_{*}(\mathrm{E})^{* *}\right)=\Sigma_{1} \leq \mathrm{i}<\mathrm{t}$ a $\mathrm{i}^{-} \mathrm{at}^{2}$ and $\mathrm{c}_{2}(\mathrm{E})-\mathrm{c}_{2}\left(\pi_{*}(\mathrm{E})^{* *}\right)=\mathrm{h}^{0}(\mathrm{Z}, Q)+\mathrm{h}^{0}\left(\mathrm{Z}, \mathrm{R}^{1} \pi_{*}(\mathrm{E})\right)$ proved in $[\mathbf{B}$, Th. 0.3] and in $[\mathbf{F M}]$ respectively. Here, of course, we assume that $E$ is extended to a compactification, however these integers do not depend upon the choices of compactification and of extension of $\mathrm{E}$.

Proof of 0.2. By [B] Th. 0.2 every admissible sequence $\left(a_{i}, b_{i}\right)$ is associated to a rank two bundle $\mathrm{E}$ on $\mathrm{W}$, moreover, the intermediate steps of the construction of $\mathrm{E}$ give bundles $E_{i}$ with splitting types $\left(a_{i}, b_{i}\right)$ for each $i$. Now use Th. 0.1 to calculate $z$ and $w$.

Remark 1.4. If we assume that $E$ has splitting type $(j,-j+\varepsilon)$ with $j \geq 1+\varepsilon$, then because $b_{1}$ $=-\mathrm{j}+\varepsilon$, we obtain $\mathrm{w} \geq \mathrm{j}-1-\varepsilon$.

Proof of 0.4. By [B] Th. 0.5 we know that (i) and (ii) are equivalent. By Lemma 1.2 (i) implies (iv). Since $\mathrm{b}_{1}=-\mathrm{j}+\varepsilon, \mathrm{b}_{\mathrm{i}}>\mathrm{b}_{\mathrm{i}-1} \forall \mathrm{i}>1$ holds, and $\mathrm{a}_{1}=\mathrm{j}$ and $\mathrm{a}_{\mathrm{i}}+\mathrm{b}_{\mathrm{i}}=\varepsilon+\mathrm{i}-1 \forall \mathrm{i}$, it follows from Theorem 0.1 that (iv) implies (ii). 
Proof of 0.5. Given bundles $\mathrm{G}$ on $\mathrm{S}$ and $\mathrm{F}$ on $\mathrm{W}$ with $\mathrm{c}_{1}(\mathrm{G})=0=\mathrm{c}_{1}(\mathrm{~F})$ there exists a bundle E on $\mathbf{S}$ satisfying $\mathrm{E}\left|\mathbf{S}-l=\pi^{*} \mathrm{E}\right| \mathrm{S}-\{\mathrm{p}\}$ and $\mathrm{E} \mid W=F$ (see [G3] Cor. 3.4).It then follows that $c_{2}(E)-c_{2}\left(\pi_{*}(E)^{* *}\right)=R^{l} \pi_{*}(F)+l(Q)$ and the result follows from Th. 0.2.

\section{Bundles of higher rank}

In this section we consider vector bundles with rank $r \geq 3$. Fix a rank $r$ vector bundle $\mathrm{E}$ on $\mathrm{U}$. We use the notation of [BG] $B 3$ for the admissible sequence $\left\{\mathrm{E}_{\mathrm{i}}\right\}, 1 \leq \mathrm{i} \leq \mathrm{t}$ of vector bundles associated to $\mathrm{E}$. In particular we denote by $(\mathrm{a}(\mathrm{i}, 1), \ldots, \mathrm{a}(\mathrm{i}, \mathrm{r}))$ the splitting type of $E_{i}$ where for $a(i, 1) \geq \ldots \geq a(i, r)$. We make the strong assumption that a(i,r-1) $\geq-1$ for every $\mathrm{i}$ and compute $\mathrm{h}^{0}\left(\mathrm{Z}, \mathrm{R}^{1} \pi_{*}(\mathrm{E})\right)$.

Proposition 2.1. Let $E$ be a rank $r$ vector bundle on $W$ whose associated sequence of vector bundles $\left\{E_{i}\right\}$ has splitting type $(a(i, 1), \ldots, a(i, r))$ with $1 \leq i \leq t$ and $a(i, r-1) \geq-1$, for all $i$. Then we have $h^{0}\left(Z, R^{l} \pi_{*}(E)\right)=\Sigma_{1 \leq i \leq t} \min \{-a(r, i)-1,0\}$.

Proof. We first observe that the proof of the corresponding inequality for rank 2 bundles works verbatim (both cases $\mathrm{t}=1$ and $\mathrm{t}>1$ ), because for each $\mathrm{i}$ with $1 \leq \mathrm{i} \leq \mathrm{t}$ at most one of the integers a $(i, j)$ is not at least -1 and $h^{1}\left(\mathbf{P}^{1}, L\right)=0$ for every line bundle $L$ on $\mathbf{P}^{1}$ with $\operatorname{deg}(\mathrm{L}) \geq-1$.

In the case $r \geq 3$, the sequence of elementary transformations made to balance the bundle is not, a priori, uniquely determined, and hence the sequence of associated bundles is not uniquely determined by $\mathrm{E}$. The condition $\mathrm{a}(1, \mathrm{r}-1) \geq-1$ implies that there is an associated sequence in which we make always an elementary transformation with respect to $\boldsymbol{O}_{\mathrm{D}}(\mathrm{a}(\mathrm{r}, \mathrm{i}))$ to pass from $\mathrm{E}_{\mathrm{i}}$ to $\mathrm{E}_{\mathrm{i}+1}$ for some a(r,i) $\leq-1$ (which gives that $\mathrm{h}^{0}\left(\mathrm{Z}, \mathrm{R}^{1} \pi_{*}\left(\mathrm{E}_{\mathrm{i}}\right)\right)=$ $\left.\mathrm{h}^{0}\left(\mathrm{Z}, \mathrm{R}^{1} \pi_{*}\left(\mathrm{E}_{\mathrm{i}+1}\right)\right)-\mathrm{a}(\mathrm{r}, \mathrm{i})+1\right)$. We continue to perform elementary transformations until we arrive at an integer $m \leq t$ such that $a(m, j) \geq-1$ for every $i$. It is then quite easy to check that $\mathrm{h}^{0}\left(\mathrm{Z}, \mathrm{R}^{1} \pi_{*}\left(\mathrm{E}_{\mathrm{m}}\right)\right)=0$ and the result follows.

In the general case the same method gives the following partial result.

Proposition 2.2. Let $E$ be a rank $r$ vector bundle on $W$ whose associated sequence $\left\{E_{i}\right\}$, $1 \leq i \leq t$ of vector bundles has splitting type $(a(i, 1), \ldots, a(i, r))$ with $1 \leq i \leq t$. Then we have $h^{0}\left(Z, R^{l} \pi_{*}(E)\right) \leq \Sigma_{1 \leq i \leq t, 1 \leq j \leq r} \min \{-a(j, i)-1,0\}$.

\section{References}

[B] E. Ballico, Rank 2 vector bundles in a neighborhood of an exceptional curve of a smooth surface, Rocky Mountain J. Math., 29 (1999), 1185-1193. 
[BG1] E. Ballico, E. Gasparim, Vector bundles on a formal neighborhood of a curve in a surface, Rocky Mountains J. Math. 30, n. 3 (2000), 1-20.

[BG2] E. Ballico, E. Gasparim, Numerical invariants for bundles on blow-ups, Proc. of the AMS, to appear

[Bu] N. P. Buchdahl, Blow-ups and gauge fields, alg-geom/9505006

[FM] R. Friedman and J. Morgan, On the diffeomorphism type of certain algebraic surfaces, II, J. Diff. Geometry 27 (1988), 371-398.

[G1] E. Gasparim, Holomorphic vector bundles on the blow-up of $\boldsymbol{C}^{2}$, J. Algebra 199 (1998), 581-590.

[G2] E. Gasparim, Chern classes of bundles on blown-up surfaces, Comm. Algebra, Vol. 28, n.10,(2000) 4912-4926.

[M] M. Maruyama, On a generalization of elementary transformations of algebraic vector bundles, Rend. Sem. Mat. Univ. Politec. Torino (1987), 1-13.

Edoardo Ballico

Dept. of Mathematics, University of Trento

38050 Povo (TN) - Italy

fa0: 39-0461881624

e-mail: ballico@science.unitn.it

Elizabeth Gasparim

Department of Mathematics

University of Texas at Austin

Austin TX 78712

e-mail: gasparim@math.utexas.edu 